\title{
Genetic divergence under uniform selection. III. Selection for knockdown resistance to ethanol in Drosophila pseudoobscura populations and their replicate lines
}

\author{
Ary A. Hoffmann* $*^{\ddagger}$ and \\ Frederick M. Cohan* $\dagger \S$
}

* Department of Genetics, University of California,
Davis, CA 95616 .
+ Department of Ecology and Evolutionary Biology,
University of Arizona, Tucson, AZ 85721 .

Replicate $D$. pseudoobscura lines from populations collected at different geographic locations were selected for increased knockdown resistance to ethanol. Population background affected the initial rate of response but not the extent that lines responded. Lines were tested for physiological traits contributing to increased knockdown resistance. Populations showed different correlated responses for two traits (tolerance of ethanol, and of acetone), suggesting that they had responded to selection by different mechanisms. Replicate lines had diverged for most traits. The results indicate that drift and/or differences in genetic background can lead to divergence under uniform selection, even when fairly large population sizes are maintained.

\section{INTRODUCTION}

Phenotypic divergence in quantitative traits among conspecific populations and closely related species is often interpreted as the result of selection in different environments. However, the same selection pressures can lead to diverse responses among populations. Genetic differences among populations before selection may affect their subsequent response. In addition, population divergence may arise as a consequence of genetic drift during the course of selection. Both these factors have been documented in artificial selection experiments with Drosophila melanogaster. The influence of genetic background is evidenced by the divergent responses of isofemale strains to selection for scutellar bristle number (Hosgood and Parsons, 1967) and crossveinlessness (Milkman, 1979), and by the effect of population background on selection for sternopleural bristles (Lopez-Fanjul and Hill, 1973) and ethanol tolerance (Gibson et al., 1979). Examples of random drift among lines with the same initial genetic background include selection for abdominal bristle number (Jones et al., 1968;

Present addresses: $\ddagger$ Department of Genetics and Human Variation, La Trobe University, Bundoora, Victoria, 3083 Australia. $\S$ Department of Biology, Wesleyan University, Middletown, CT 06457, U.S.A.
Yoo, 1980) and modification of the expression of cubitus interruptus (Cohan, 1984a). Unfortunately, it is difficult to relate many of these studies to the effect of genetic drift on natural populations undergoing selection. This is because only a few individuals were selected in each generation, whereas the effective population size of natural populations may be several hundred (Wright, 1978).

To investigate the effects of genetic drift and population background in fairly large populations, Cohan and Graf (1985) and Cohan and Hoffmann (1986) selected for knockdown resistance to ethanol in D. melanogaster. Two replicate lines from each of five geographically separate populations were selected and two other lines were maintained as unselected controls. Before selection the populations showed a weak latitudinal cline for knockdown resistance. This cline was steepened after 24 generations of selection, indicating that initial genetic differences among populations affected the selection responses.

The lines were scored for physiological and behavioural traits to investigate possible differences in the mechanisms whereby selected lines had become more resistant to knockdown by ethanol (Cohan and Hoffmann, 1986). Several of the traits showed small effects of genetic background 
(geographic origin) on the selection response. However, there were much larger differences in response among the replicate lines, indicating that genetic drift led to divergence despite an effective population size of 154 . Drift caused more divergence among selected replicates than control replicates, indicating that its effect was accentuated by selection in accordance with a theoretical analysis by Cohan (1984b). Population background did have a marked effect on productivity (number of emerging progeny), which was scorẹd to examine effects of selection on a fitness component.

In this paper we consider genetic divergence in another species, $D$. pseudoobscura, which has a range that partly overlaps with $D$. melanogaster in North America. Several studies have demonstrated geographic differences among $D$. pseudoobscura populations for morphological and physiological traits (e.g., Anderson, 1968; Coyne et al., 1983), as have numerous studies with $D$. melanogaster (Parsons, 1982). These differences are expressed under constant conditions and therefore genetically based, and indicate the potential for genetic background effects on selection responses. We describe the response to selection and present evidence for effects of population background and genetic drift on the mechanisms of the response to selection.

\section{MATERIALS AND METHODS}

Most of the methods follow Cohan and Graf (1985) and Cohan and Hoffmann (1986), and only brief descriptions are provided here.

\section{Establishment of lines}

In the summer of 1982 samples of wild $D$. pseudoobscura were collected from five localities on the west coast of North America: Oxnard, CA $\left(\mathrm{OX}, 34^{\circ} \mathrm{N}\right)$; Watsonville, $\mathrm{CA}\left(\mathrm{WA}, 37^{\circ} \mathrm{N}\right)$; Camino, $\mathrm{CA}\left(\mathrm{CA}, 39^{\circ} \mathrm{N}\right)$; Kerby, OR $\left(\mathrm{KE}, 42^{\circ} \mathrm{N}\right)$; and Port Coquitlam, British Columbia $\left(\mathrm{PC}, 49^{\circ} \mathrm{N}\right)$. These localities range from Southern California to British Columbia, spanning approximately the same latitudinal transect as the $D$. melanogaster study cited above. D. pseudoobscura flies were distinguished cytologically or electrophoretically by A. Beckenbach and L. Barr.

Each population was started with 12 isofemale strains, 3-6 months after the strains had been collected. To eliminate effects of multiple mating on genetic variation, each isofemale strain was rep- resented by the progeny of a male mated to a virgin female. The populations _were divided into two replicate lines to be selected (lines 1 and 2) and two replicate lines to remain unselected (lines 3 and 4). Because of low productivity, only one selected line was set up for the KE population. A second line was set up three generations later using flies from an unselected line. Each line was cultured in eight half-pint bottles at $20 \cdot 6^{\circ} \mathrm{C}$, with 32 adults (unsexed) used as founders for each bottle.

An additional unselected control $\left(C_{p}\right)$ was set up from the CA lines, and was usually tested twice on every day of selection to control for day-to-day fluctuations in knockdown resistance (see below).

\section{Selection for knockdown resistance}

Progeny were transferred to bottles with laboratory medium 25-30 days after the parents were set up. They were kept on this medium for 2 days, and all eight bottles from a line were then pooled before selecting for knockdown resistance to ethanol fumes.

The selection procedure follows Cohan and Graf (1985). Each generation 1.6-2.0 g of flies were placed in a vertical glass tube nearly saturated with ethanol fumes (except as noted below). Flies which succumbed to fumes rolled down a series of baffles to a removable collecting dish which was replaced every minute. Flies were counted (unsexed) every minute to yield a distribution of knockdown times. The last quartile to fall was kept as parents for the next generation.

Population size was enforced at 256 flies per line, except when the required number of progeny for selection $(4 \times 256)$ was not available. This led to the following harmonic means of census sizes for the selected lines over 18 generations of selection: OX-1, 167; OX-2, 214; CA-1, 156; CA-2, 218; WA-1, 220; WA-2, 182; KE-1, 160; KE-2, 153 (over 15 generations); PC-1, 126 and PC-2, 156. Hence the overall harmonic means of the selected and control lines are 175 and 256 respectively.

Data from each knockdown trial were summarised as the mean time taken for flies to fall down the tube (mean knockdown time). Using the mean was considered appropriate because the knockdown times for flies were approximately normally distributed. Means are also useful in heritability estimates, because the realised heritability from a selection response based on means can be related to heritability in the base population.

Lines were kept for the first 15 generations at U.C. Davis and a further five generations at the University of Arizona. Selection was not carried 
out in generations 14,16 and 17 . The laboratory medium at Davis contained cornmeal, semolina, dextrose, sucrose, dried yeast and agar, while the Arizona medium contained bananas, dried yeast, malt, corn syrup and agar. Both media had propionic acid as a preservative. This change in medium may have affected knockdown resistance as knockdown times for line $C_{p}$ and the selected lines were lower in Arizona than in Davis. (The mean knockdown time of line $C_{p}$ was 14.82 minutes in Davis and 12.98 minutes in Arizona $[t=1 \cdot 40,16 \mathrm{df}, P<0 \cdot 10]$; the mean knockdown time of selected lines was 19.95 minutes for the last three generations at Davis and $17 \cdot 27$ minutes for the three generations at Arizona $[t=4.35$ for paired test, $9 \mathrm{df}, P<0.01]$ ).

\section{Correction of selection response}

Fluctuations in the $C_{p}$ line tended to follow those of the selection lines. Investigators often subtract the control phenotype from that of the selected line to remove effects of environmental variation and natural selection. This procedure can lead to overcorrection if control and selected lines are differentially responsive to some agent of phenotypic change. We therefore used a multiple regression method (Cohan and Harshman, unpubl.) to empirically estimate the fraction by which changes in selected lines should be corrected by changes in control lines. For each selected line, the selected phenotype $\left(s_{i}\right)$ in each generation $(i)$ was modelled as a polynomial function of the generation of selection and the phenotype of the control $\left(c_{i}\right)$ in each generation: $s_{i}=$ $b_{1} i+b_{2} i^{2}+b_{3} c_{i}+a+E$, in which $a$ is the intecept, $E$ the error term, and the $b_{j}$ 's the partial regression coefficients. The quadratic term for $i$ was included in the preliminary analysis of each line to allow for attenuation of selection response over time; the term was kept when it was significant at $P<$ 0.20 (lines OX-1, WA-1, WA-2, and CA-1). The partial coefficient for the controls $\left(b_{3}\right)$ gives the proportion by which changes in the controls are reflected by changes in the selected phenotype. The selected line phenotypes were then corrected as: $s_{i}=s_{i}-b_{3} c_{i}+c$, in which $s_{i}$ is the corrected phenotype for generation $i$ and $c$, is the grand mean knockdown time of the control over all generations. Correction factors ranged from 0.28 to 0.94 with a mean of 0.60 . This procedure was only applied to the 15 generations of selection at Davis because the relationship between fluctuations in selected and control lines may have changed with the medium.

\section{Single day measurements of knockdown resistance}

To accurately examine the selection responses, all 20 selected and unselected lines were measured for knockdown resistance in two complete randomised blocks of continuous testing after 18 generations of selection. Flies were cultured as for selection except that three bottles were used per line (Arizona medium) and the knockdown resistance of each sex was scored separately.

\section{Longevity experiments in sealed vials}

Flies were cultured on the Davis medium and were pooled across three culture bottles per line. Males were sorted by aspiration into groups of 10 and kept in vials with medium for 2 days. They were then transferred to an empty vial, covered with cheesecloth, inverted and positioned on top of another vial containing rayon and $10 \mathrm{ml}$ of water or a test solution. These vials were sealed together and the number of dead flies counted at 12 hour intervals until at least half the flies had died. The 50 per cent lethal time in hours (LT50) was linearly interpolated.

\section{Longevity experiments in hydration chambers}

Flies were cultured and prepared as for the sealed vial tests, and placed in empty vials covered with cheesecloth. These were randomised and placed in glass hydration chambers containing the test solutions. LT50s were determined for the 0 per cent, 5 per cent and 10 per cent ethanol tests by scoring mortality at 12 hour intervals. For the 20 per cent ethanol, acetone ( 1.5 per cent $)$ and acetaldehyde $(2.0$ per cent $)$, tests the vials were removed from hydration chambers after 4,24 , and 1.5 hours respectively. All flies had been knocked down by these times. Flies were thereafter transferred to vials with the Davis medium and left to recover for 24 hours before mortality was scored.

\section{Body weight and early productivity}

Flies to be weighed were collected from culture bottles and sexed under ether anaesthesia. Eight replicates of five males and five females from each line were placed in empty vials. These were randomised on a tray and dried for 24 hours at $40^{\circ} \mathrm{C}$ before weighing.

For the productivity tests, 1-day-old flies from pooled bottles were sorted by aspiration into groups of three males and three females with eight 
replicate groups per line. These were aged for 1 day in vials with the Davis medium and then transferred to vials with fresh medium every day for the next 5 days. Dead flies were replaced by identically maintained extras. Productivity was scored as. the number of adults emerging from the vials after 3 weeks incubation at $21-23^{\circ} \mathrm{C}$.

\section{RESULTS}

\section{Realised heritability estimates}

Realised heritabilities were estimated from corrected values for each line over successive fourgeneration intervals, by dividing the cumulative selection reponse by the cumulative selection differential (Falconer, 1981, Ch. 11). For the first four generations the average heritability is $0 \cdot 151 \pm$ 0.031 (table 1). An analysis of variance (ANOVA) showed significant differences among populations $(P<0.01)$, with 88.0 per cent of the variation in heritability attributed to populations. Heritabilities tended to decrease over the next two four-generation periods and increase again in the last period (table 1). Populations showed no significant differences in realised heritability after the first four generations, or over all generations pooled. The initially higher heritabilities of some populations were therefore not maintained during selection.

\section{Responses over fifteen generations of selection}

A quadratic regression of the corrected mean knockdown times on generation of selection was carried out for each selected line, with the quadratic term removed when it was not significant (at $P<0 \cdot 20$ ). These regressions included only the first 15 rounds of selection because of the possible decrease in knockdown times due to culturing on banana medium. The predicted values of knockdown times before and after selection are given in table 2. The expected value for KE-2 was calculated by linear extrapolation from the first 12 rounds of selection. The average predicted value before selection was $13 \cdot 24$ minutes compared with 20.57 minutes after selection. In contrast to the early differences in the selection responses of populations (above), ANOVAs indicate no significant population differences in predicted values before or after 14 generations of selection, or in the amount of change over the course of selection.

A linear regression of predicted values before selection on latitude indicates a marginally significant cline of increasing knockdown time in higher latitudes $(b=0 \cdot 16 \pm 0.05$ minutes knockdown time per degree latitude, $3 \mathrm{df}, P=0.06$ ); the cline persisted after 14 generations with little change in slope $(b=0 \cdot 18 \pm 0.02, \quad P=0.002)$. Latitudinal clines in knockdown times have also been observed among selected and unselected populations of D. melanogaster (Cohan and Hoffmann, 1986).

\section{Single-day measurements of knockdown resistance}

To compare the selection responses precisely, all selection and control lines were tested on a single day on two occasions, after 18 generations of selection. The mean knockdown times of selected and

Table 1 Realised heritabilities at different generations of selection. Standard errors are based on the variance among the five population means

\begin{tabular}{llrrr}
\hline & \multicolumn{2}{l}{ Realised heritability } & \multicolumn{1}{l}{} \\
Selected line & $1-4$ & $5-8$ & \multicolumn{1}{l}{-12 } & \multicolumn{1}{l}{$13-14$} \\
\hline OX-1 & 0.227 & 0.010 & 0.084 & -0.044 \\
OX-2 & 0.212 & -0.015 & 0.103 & 0.117 \\
WA-1 & 0.233 & 0.051 & 0.017 & 0.131 \\
WA-2 & 0.231 & 0.187 & -0.031 & 0.257 \\
CA-1 & 0.128 & 0.209 & 0.063 & -0.023 \\
CA-2 & 0.064 & 0.117 & -0.014 & 0.364 \\
KE-1 & 0.097 & 0.083 & -0.001 & 0.028 \\
KE-2 & 0.100 & 0.041 & $0.088^{*}$ & \\
PC-1 & 0.132 & 0.194 & -0.007 & 0.216 \\
PC-2 & 0.087 & 0.195 & -0.019 & 0.024 \\
Mean (S.E., $N=5)$ & $0.151(0.031)$ & $0.107(0.035)$ & $0.033(0.017)$ & $0.160(0.040)$ \\
\hline
\end{tabular}

* Realised heritability for generations 9-11. 
Table 2 Predicted values of knockdown times (minutes) at generations 0 and 14, calculated from the regression of knockdown time on generation of selection. Standard errors are based on the variance among the five population means

\begin{tabular}{lll}
\hline Selected line & Generation 0 & Generation 14 \\
\hline OX-1 & $10 \cdot 66$ & $18 \cdot 86$ \\
OX-2 & $13 \cdot 37$ & $19 \cdot 71$ \\
WA-1 & $13 \cdot 82$ & $19 \cdot 82$ \\
WA-2 & $11 \cdot 43$ & $20 \cdot 71$ \\
CA-1 & $11 \cdot 51$ & $20 \cdot 30$ \\
CA-2 & $14 \cdot 30$ & $20 \cdot 41$ \\
KE-1 & $14 \cdot 65$ & $18 \cdot 23$ \\
KE-2 & $14 \cdot 22$ & $23 \cdot 52^{*}$ \\
PC-1 & $14 \cdot 13$ & $22 \cdot 73$ \\
PC-2 & $14 \cdot 27$ & $21 \cdot 42$ \\
Mean (S.E., $N=5)$ & $13 \cdot 24(0 \cdot 47)$ & $20 \cdot 57(0 \cdot 46)$ \\
\hline
\end{tabular}

* The predicted value for KE-2 at generation 14 was linearly extrapolated from generations $0-11$.

control lines were $19.68 \pm 0.52$ and $14.60 \pm 0.25$ minutes respectively. An ANOVA on the unweighted means of the two sexes shows that 68 per cent of the variance is due to selection $(P<0 \cdot 0001)$, 6.8 per cent to replicate line $(P=0.08)$ and none to population or the interaction between population and selection. Similar results are obtained if the sexes are analysed separately. There was no significant latitudinal cline in knockdown times before or after selection as described above. This may be due to the change to banana medium or to genetic drift.

\section{Correlated responses-Longevity tests with ethanol}

LT50s were not transformed prior to analysis but proportions were arcsine transformed. The variance components and significance levels for the ANOVAs are presented in table 3. Means and standard errors given below are based on replicate means or population means (if the population term was at least marginally significant in the ANOVA).

Considering first the sealed vial tests, the mean LT50 of all lines exposed to 1 per cent ethanol $(112.4 \pm 3.5$ minutes) was higher than when only water was provided $(86 \cdot 3 \pm 1 \cdot 7$ minutes $)$. This suggests that $D$. pseudoobscura utilises 1 per cent ethanol as a metabolic resource as may other Drosophila species attracted to fermented-fruit baits (Holmes et al., 1980). The only significant effect in the ANOVAs is replicate line, so longevity in the presence of 1 per cent ethanol did not show a correlated response to selection.

The mean longevity of flies in the presence of water was lower in the hydration chamber $(74.8 \pm$ $1 \cdot 1$ minutes) than in the sealed vials $(86 \cdot 3 \pm 1 \cdot 7$ minutes), a phenomenon previously noted with $D$. melanogaster (Cohan and Hoffmann, 1986). The 5 per cent ethanol solution increased longevity

Table 3 Analyses of variance for all traits studied as correlated responses: Components of variation in percentages, with mean squares in parentheses

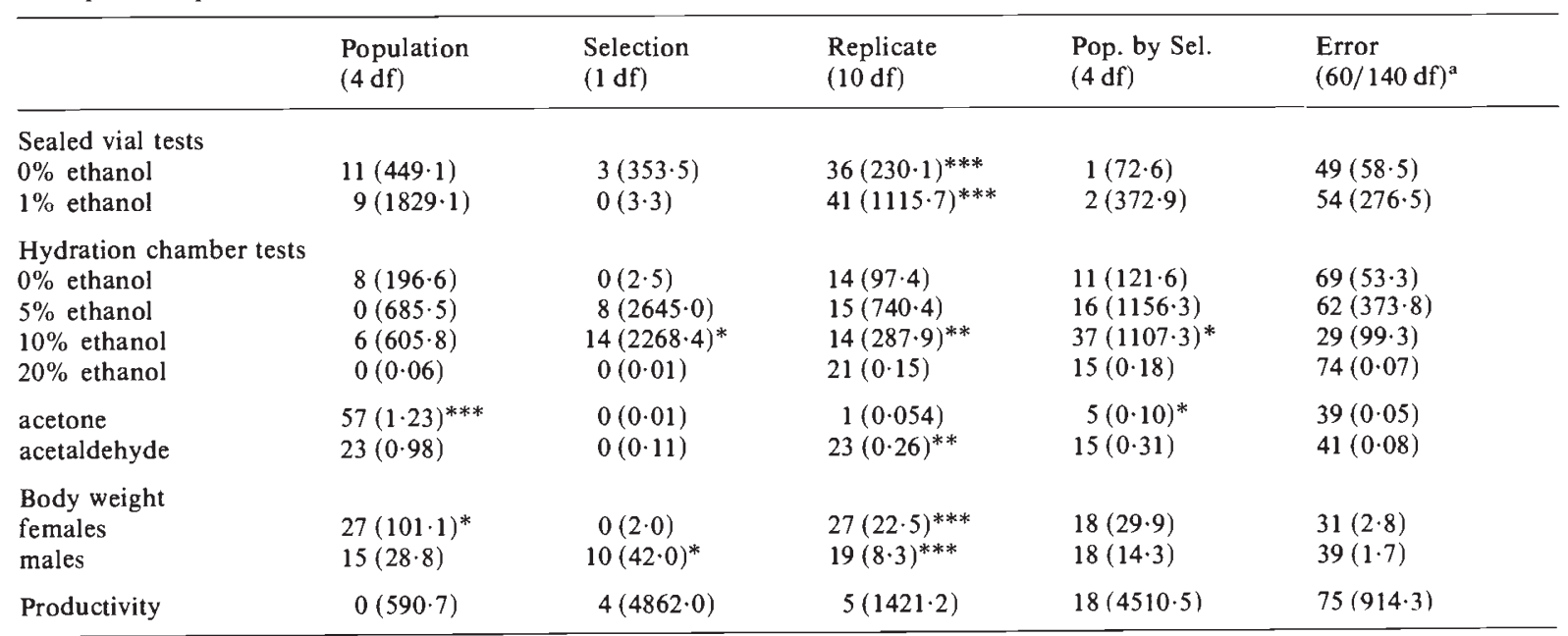

${ }^{\text {a }}$ Error term has $60 \mathrm{df}$ except for body weight and productivity where the term has $140 \mathrm{df}$.

$* P<0.05$.

** $P<0.01$.

*** $P<0.001$. 
(mean LT50 $=97 \cdot 0 \pm 3 \cdot 3$ minutes), while the 10 per cent concentration decreased longevity (table 4). A significant selection effect was only found for 10 per cent ethanol (table 3 ). Selection lines were more tolerant of this concentration than unselected lines (table 4) as predicted from knockdown resistance. The significant interaction between population and selection indicates that the magnitude of this correlated response was population dependent. For the KE, OX and PC populations both replicate selected lines were relatively more tolerant than both control lines (table 4). However, replicate lines from the WA and CA populations behaved inconsistently, which is reflected in the significance of the nested replicate line factor. None of the terms in the ANOVAs was significant for the other ethanol-related traits, including longevity under 20 per cent ethanol. The mean proportion of flies surviving after exposure to 20 per cent ethanol was similar for the selected $(0.40 \pm 0.05)$ and unselected $(0 \cdot 37 \pm 0 \cdot 04)$ lines. Hence selection for knockdown resistance to ethanol led to a concentration-specific increase in longevity.

\section{Correlated responses-other traits}

The tolerances of lines to acetaldehyde ( 2 per cent) and acetone ( 1.5 per cent) was also tested. Acetaldehyde is metabolically related to ethanol, being an intermediate in its breakdown, and is utilised as a resource at low concentrations in several Drosophila species (Parsons and Spence, 1981). Acetone is not directly related to ethanol metabolism and is not utilised by D. melanogaster (Van Herrewege et al., 1980), so provides a possible test for non-metabolic mechanisms of knockdown resistance. Survival in the presence of acetaldehyde and acetone was not correlated with the response to selection (table 3). However, the effects of selection on survival with acetone were different across populations, as indicated by a significant population by selection interaction. Line means (table 4) indicate that mortality is lower for the selected lines from the WA and PC populations, suggesting a correlated response to selection in these populations. No effect was evident in the KE and CA populations, while selected lines from the OX population were less tolerant of this chemical than the control lines. Populations differed overall in their tolerances of both acetone and acetaldehyde.

Body weight showed a correlated response to selection in males but not in females. Males from the selected lines were lighter overall (table 4), but the body weight of females did not show the same trend (mean weight of five flies from selected lines,
$0.00214 \pm 0.00008 \mathrm{~g}$; mean weight of control lines, $0.00217 \pm 0.00009 \mathrm{~g})$. There are also significant replicate line effects for both traits, and a significant population effect for female body weight. In the unselected lines, flies from the northern populations were heavier than those from the southern populations, as indicated by a linear regression of body weight on latitude (males, $b=0 \cdot 15 \pm 0.05$, $P<0.05$; females, $b=0 \cdot 24 \pm 0 \cdot 13$, NS). A latitudinal cline for body weight in $D$. pseudoobscura has previously been described by Sokoloff (1966).

The ANOVA on productivity indicates that there are no significant differences among lines, although both the selection and selection by population interaction terms are marginally significant $(P<0 \cdot 10)$. Two of the populations (WA, KE) appear to have selection lines with lower productivities whereas productivities of the other populations seem to be unaffected by selection (table 4).

\section{DISCUSSION}

The heritability estimates indicate that populations responded to selection at different rates in the early generations. However, they did not differ significantly in their responses over the entire course of selection. A possible explanation is that the genes involved were at low to intermediate frequencies initially in all populations, and that there were differences in gene frequencies among populations. The response to selection is proportional to the allele frequency product (Crow and Kimura, 1970, Ch. 5), so populations with intermediate frequencies of resistance alleles would show a rapid response initially, follwed by a slower response (as may have occurred in the OX population). In contrast, populations with low frequencies of resistance alleles would respond slowly initially and more rapidly as the allele frequencies become intermediate (as may have occurred in the KE-1 line). The same genes need not be involved in different populations, as long as they have a comparable effect on knockdown resistance.

Although the $D$. pseudoobscura populations began selection with the same realized heritability as D. melanogaster $\left(h^{2}=0 \cdot 143\right.$; Cohan and Graf, 1985), they responded much more slowly to selection. The $D$. pseudoobscura populations responded on average only 0.52 minutes per generation, compared to $1 \cdot 10$ for $D$. melanogaster (Cohan and Hoffmann, 1986). The difference is due to a greater reduction of heritability over the course of selection in $D$. pseudoobscura (average realised heritability estimated over all generations is 
Table 4 Line means (and standard deviations) for tolerance of 10 per cent ethanol (hydration chamber) and acetone, male body weight and productivity

\begin{tabular}{|c|c|c|c|c|}
\hline Lines & $\begin{array}{l}10 \% \text { ethanol } \\
\text { (LT 50) }\end{array}$ & $\begin{array}{l}\text { Acetone } \\
\text { (\% survival) }\end{array}$ & $\begin{array}{l}\text { Male body weight } \\
\left(\mathrm{g} \times 10^{-4}\right)\end{array}$ & $\begin{array}{l}\text { Productivity } \\
\text { (no. of progeny) }\end{array}$ \\
\hline \multicolumn{5}{|l|}{ Selected } \\
\hline OX-1 & $67 \cdot 4(8 \cdot 2)$ & $14(4)$ & $18 \cdot 5(1 \cdot 1)$ & $69 \cdot 0(27 \cdot 8)$ \\
\hline$O X-2$ & $58 \cdot 8(8 \cdot 3)$ & $29(17)$ & $16 \cdot 4(0.9)$ & $102 \cdot 5(24 \cdot 5)$ \\
\hline WA-1 & $54.6(8.9)$ & $52(20)$ & $14 \cdot 0(0 \cdot 8)$ & $80 \cdot 4(14 \cdot 6)$ \\
\hline WA-2 & $53 \cdot 1(9 \cdot 6)$ & $63(12)$ & $14 \cdot 4(1 \cdot 2)$ & $84 \cdot 1(35 \cdot 2)$ \\
\hline CA-1 & $54 \cdot 9(3 \cdot 2)$ & $19(14)$ & $16 \cdot 2(1 \cdot 0)$ & $98 \cdot 5(29 \cdot 9)$ \\
\hline CA-2 & $40 \cdot 9(2 \cdot 2)$ & $5(6)$ & $14 \cdot 7(1 \cdot 0)$ & $117 \cdot 7(36 \cdot 9)$ \\
\hline KE-1 & $52 \cdot 5(9.9)$ & $57(11)$ & $16 \cdot 1(1 \cdot 4)$ & $73 \cdot 6(41 \cdot 4)$ \\
\hline $\mathrm{KE}-2$ & $61 \cdot 6(15 \cdot 0)$ & $29(14)$ & $17 \cdot 5(1 \cdot 4)$ & $68 \cdot 1(16 \cdot 1)$ \\
\hline PC-1 & $42 \cdot 8(9 \cdot 3)$ & $78(26)$ & $17 \cdot 6(0.9)$ & $100 \cdot 6(42 \cdot 4)$ \\
\hline $\mathrm{PC}-2$ & $26 \cdot 0(4 \cdot 5)$ & $71(23)$ & $15 \cdot 0(0 \cdot 7)$ & $84 \cdot 7(29 \cdot 8)$ \\
\hline
\end{tabular}

Unselected

$\begin{array}{llllr}\text { OX-3 } & 42 \cdot 9(3 \cdot 8) & 76(13) & 16 \cdot 4(1 \cdot 1) & 87 \cdot 2(28 \cdot 6) \\ \text { OX-4 } & 40 \cdot 6(8 \cdot 4) & 58(3) & 15 \cdot 9(1 \cdot 2) & 111 \cdot 2(21 \cdot 8) \\ \text { WA-3 } & 23 \cdot 9(9 \cdot 6) & 49(18) & 15 \cdot 6(0 \cdot 7) & 114 \cdot 9(27 \cdot 1) \\ \text { WA-4 } & 30 \cdot 3(13 \cdot 7) & 32(17) & 16 \cdot 7(2 \cdot 8) & 94 \cdot 2(33 \cdot 7) \\ \text { CA-3 } & 36 \cdot 8(5 \cdot 8) & 14(7) & 17 \cdot 1(1 \cdot 1) & 83 \cdot 2(22 \cdot 8) \\ \text { CA-4 } & 33 \cdot 1(1 \cdot 9) & 10(14) & 16 \cdot 1(0 \cdot 8) & 101 \cdot 9(25 \cdot 0) \\ \text { KE-3 } & 60 \cdot 9(11 \cdot 8) & 28(17) & 17 \cdot 7(1 \cdot 9) & 109 \cdot 0(46 \cdot 4) \\ \text { KE-4 } & 36 \cdot 4(18 \cdot 4) & 45(13) & 18 \cdot 9(1 \cdot 1) & 119 \cdot 2(34 \cdot 2) \\ \text { PC-3 } & 54 \cdot 7(18 \cdot 6) & 44(17) & 17 \cdot 6(1 \cdot 3) & 75 \cdot 5(19 \cdot 6) \\ \text { PC-4 } & 44 \cdot 7(10 \cdot 3) & 39(20) & 18 \cdot 4(1 \cdot 5) & 93 \cdot 1(23 \cdot 0)\end{array}$

Means and standard deviations are based on four (tolerance) or eight (body weight, productivity), replicates.

$0.130 \pm 0.009$ for D. melanogaster; Cohan and Hoffmann, unpubl.) and to lower phenotypic variance in $D$. pseudoobscura (average phenotypic standard deviation of $5 \cdot 40 \pm 0 \cdot 029$ minutes for $D$. pseudoobscura and $7 \cdot 42 \pm 0.025$ minutes for $D$. melanogaster, based on first four generations; Cohan, unpubl.).

Population background affected responses in ethanol tolerance ( 10 per cent hydration chamber) and acetone tolerance. The differences between populations were marked, with many populations showing no correlated responses for these traits. As all populations had the same knockdown resistance after selection, the different correlated responses indicate that they may have responded to selection by different mechanisms. The ethanol data indicate that the increased knockdown resistance in the OX, WA and CA populations may be associated with the metabolic breakdown of ethanol. The acetone results suggest that a decreased rate of entry of ethanol may have been involved in the WA and PC populations.

Other mechanisms of knockdown resistance not measured by ethanol and acetone tolerance are also involved because the OX population did not show a correlated response for either of these traits, but reponded to selection to the same extent as the other populations. Possibilities include clinging to baffles or short-term closure of spiracles to prevent entry of ethanol fumes. Genes affecting male body size also seem to be involved, perhaps by decreasing fall rate in the knockdown tube, although it is not clear why this effect should be sex-specific.

These findings differ from the D. melanogaster results described by Cohan and Hoffmann (1986). In $D$. melanogaster, the metabolic breakdown of ethanol seems to have contributed to the response to selection for knockdown resistance. Lines selected for increased knockdown resistance were more tolerant than control lines of 17 per cent and 20 per cent ethanol in the hydration chamber, and were also more tolerant of the metabolically related chemical acetaldehyde. In contrast, ethanol metabolism may have contributed little to the response to selection in $D$. pseudoobscura. Only some selected lines were more tolerant of 10 per cent ethanol and there was no correlated response at the higher 
concentration of ethanol or for tolerance of acetaldehyde.

The effects of population background on mechanisms of the selection response also contrast with the $D$. melanogaster experiments, where all lines showed the same correlated responses and there were only small quantitative effects of population background. Perhaps the $D$. melanogaster populations were more similar genetically, although this seems unlikely given the vagility of $D$. pseudoobscura in some mark-release-capture experiments (Jones et al., 1981; Coyne et al., 1982) and the greater degree of population structure as inferred from allozyme frequencies in $D$. melanogaster (Wright, 1978).

Initial genetic differences may lead to different selection responses under an epistatic or non-epistatic model of fitness. The non-epistatic model assumes that all resistance alleles are favoured in all genetic backgrounds. Populations will differ in their response if they differ in genetic variation at some of the loci. They are then not likely to diverge if there is gene flow of favoured alleles between populations during selection. In the epistatic model, some alleles favoured in the genetic background of one population are not favoured in the background of another (Wright, 1970). Populations may then diverge even with moderate gene flow.

Both models help to explain divergence among the $D$. pseudoobscura populations. The ethanol tolerance results are consistent with a non-epistatic model. Populations showing an increased tolerance of ethanol after selection had lower tolerances prior to selection (table 4). Populations with higher initial tolerance may not show increased ethanol tolerance because there was no remaining genetic variation for this trait. The acetone results are more consistent with an epistatic model. Acetone tolerance increased with selection in two populations (WA, PC) while another (OX) showed a decrease (table 4). A higher level of tolerance may have conferred more resistance to knockdown by ethanol in the WA and PC backgrounds while the same alleles may have decreased resistance in the OX background. An alternative explanation not requiring epistasis is that the populations were segregating different acetone tolerance alleles, each with a different pleiotropic effect on ethanol knockdown resistance.

These models can be distinguished by introducing knockdown resistance factors from one population into the genetic backgrounds of other populations. For example, the epistatic model predicts that the F1s and F2s of crosses between selected lines from different populations should show a loss of the selected phenotype (e.g., King, 1955). Unfortunately the small differences in knockdown resistance between selected and control $D$. pseudoobscura lines makes this difficult to test. Responses to selection in D. melanogaster were greater (Cohan and Hoffmann, 1986), and crosses between different populations of this species gave no support to the epistatic model (Cohan, Gayley and Hoffmann, unpubl.).

Replicate lines diverged for most traits, indicating that genetic drift occurred. We did not estimate the effective population size of the $D$. pseudoobscura lines, although an estimate for similarity maintained $D$. melanogaster lines was 60 per cent of the census size (Cohan and Hoffmann, 1986). The effective population size of $D$. pseudoobscura was probably less than in $D$. melanogaster because of the smaller harmonic mean of the selected lines and the lower number of progeny emerging from culture bottles each generation. This may explain why fewer $D$. melanogaster traits showed significant replicate line effects. Genetic divergence can be greater among selected replicate lines than unselected replicates because of a lower effective population size or an increased probability of fixing alternative alleles (Cohan, 1984b). Cohan and Hoffmann (1986) presented evidence for the latter mechanism in D. melanogaster. A similar analysis was not possible in $D$. pseudoobscura because few of the traits showed a response to selection, and only one of these (10 per cent ethanol) had significant replicate line divergence. However, the replicate line term is no longer significant $\left(\mathrm{F}_{(6 \cdot 36)}=2 \cdot 10\right)$ if the two populations ( $\mathrm{OX}$, CA) not showing a correlated response for this trait are discarded.

In conclusion, we have found that population background and drift may affect the rate and mechanism of response to a similar selection pressure, so lines can reach different genetic endpoints after selection. Phenotypic divergence among conspecific populations can therefore arise without evolutionary responses to different environments, even when the census population size is fairly large. This type of divergence may help to explain the divergent responses of populations of various species to environmental stresses (review, Cohan 1984b).

Acknowledgements We thank Larry Harshman, Michael Turelli and Peter Parsons for comments on an earlier version of this paper. We thank Tim Prout, Conrad Istock, William Heed and Richard Michod for use of facilities. This research was supported by National Institutes of Health grant GM22221 and National Research Service Award GM08511. 


\section{REFERENCES}

ANDERSON, W. W. 1968. Further evidence for coadaptation in crosses between geographic populations of Drosophila pseudoobscura. Genet. Res., 12, 317-330.

COHAN, F. M. 1984a. Genetic divergence under uniform selection. I. Similarity among populations of Drosophila melanogaster in their response to artificial selection for modifiers of $c i^{D}$. Evolution, 38, 55-71.

COHAN, F. M. 1984b. Can uniform selection retard random genetic divergence between isolated conspecific populations? Evolution, 38, 495-504.

COHAN, F. M. AND GRAF, J. 1985. Latitudinal cline in Drosophila melanogaster for knockdown resistance to ethanol fumes and for rates of response to selection for further resistance. Evolution, 39, 278-293.

COHAN, F. M. AND HOFFMANN, A. A. 1986. Genetic divergence under uniform selection. II. Different responses to selection for knockdown resistance to ethanol among Drosophila melanogaster populations and their replicate lines. Genetics, (in press).

COYNE, J. A., BOUSSY, I. A., PROUT, T., BRYANT, S. H., JONES, J. S. AND MOORE, J. A. 1982. Long-distance migration of Drosophila. Amer. Nat., 119, 589-595.

COYNE, J. A., BUNDGAARD, J. AND PROUT, T. 1983. Geographic variation of tolerance to environmental stress in Drosophila pseudoobscura. Amer. Nat., 122, 474-488.

CROW, J. F. AND KIMURA, M. 1970. An Introduction to Population Genetics Theory, Burgess, Minneapolis.

FALCONER, D. S. 1981. Introduction to Quantitative Genetics, 2nd ed., Longman, London.

GIBSON, J. B., LEWIS, N., ADENA, M. A. AND WILSON, S. R. 1979. Selection for ethanol tolerance in two populations of Drosophila melanogaster segregating $\mathrm{ADH}$ allozymes. Aust. J. Biol. Sci., 32, 387-398.

HOSGOOD, S. M. W. AND PARSONS, P. A. 1967. The exploitation of genetic heterogeneity among founders of laboratory populations of Drosophila prior to directional selection. Experientia, 23, 1066.
JONES, J. S., BRYANT, S. H., LEWONTIN, R. C., MOORE, J. A. AND PROUT, T. 1981. Gene flow and the geographical distribution of a molecular polymorphism in Drosophila pseudoobscura. Genetics, 98, 157-178.

JONES, L. P., FRANKHAM, R. AND BARKER, J. S. F. 1968. The effects of population size and selection intensity in selection for a quantitative character in Drosophila. II. Long-term response to selection. Genet. Res., 12, 249-266.

KING, J. C. 1955. Evidence for the integration of the gene pool from studies of DDT resistance in Drosophila. Cold Spring Harb. Symp. Quant. Biol., 20, 311-317.

LOPEZ-FANJUL, C. AND HILL, W. G. 1973. Genetic differences between populations of Drosophila melanogaster for a quantitative trait. I. Laboratory populations. Genet. Res., 22, 51-68.

Milkman, R. D. 1979. The posterior crossvein in Drosophila as a model phenotype. In Thompson, J. N. and Thoday, J. M. (eds.) Quantitative Genetic Variation, Academic Press, New York.

PARSONS, P. A. 1982. Adaptive strategies of colonizing animal species. Biol. Rev., 57, 117-148.

PARSONS, P. A. AND SPENCE, G. E. 1981. Acetaldehyde: a low-concentration resource and larval attractant in three Drosophila species. Experientia, 37, 576-577.

SOKOLOFF, A. A. 1966. Morphological variation in natural and experimental populations of Drosophila pseudoobscura and D. persimilis. Evolution, 20, 49-71.

VAN HERREWEGE, J., DAVID, J. R. AND GRANTHAM, R. 1980, Dietary utilisation of aliphatic alcohols by Drosophila. Experientia, 36, 846-847.

WRIGHT, S. 1970. Random drift and the shifting balance theory of evolution. In Kojima K. (ed.) Mathematical Topics in Population Genetics, Springer-Verlag, Berlin.

WRIGHT, S. 1978. Evolution and the Genetics of Populations, Vol. 4. Univ. of Chicago, Chicago.

YOO, B. H. 1980. Long-term selection for a quantitative character in large replicate populations of Drosophila melanogaster. 1. Response to selection. Genet. Res., 35, 1-17. 